\title{
Anterior Clinoid Metastasis as First Presentation of a Signet Ring Cell Carcinoma: An Intriguing Diagnosis
}

\author{
Elena d'Avella1® \\ Elia Guadagno ${ }^{2}$ \\ ${ }^{1}$ Department of Neurosurgery, Università degli Studi di Napoli \\ "Federico II," Naples, Italy \\ 2 Pathology Unit, Department of Advanced Biomedical Sciences, \\ Università degli Studi di Napoli “Federico II," Naples, Italy \\ ${ }^{3}$ Department of Advanced Biomedical Sciences, Università degli Studi \\ di Napoli “Federico II," Naples, Italy
}

\author{
Domenico Solari $^{1}$ Luigi Maria Cavallo ${ }^{1}$
}

J Neurol Surg Rep 2020;81:e46-e51.

\author{
Address for correspondence Elena d'Avella, PhD, Division of \\ Neurosurgery, Università degli Studi di Napoli “Federico II,", \\ Naples 80144, Italy (e-mail: elenadavella@gmail.com).
}

\begin{abstract}
Keywords

- anterior clinoid process

- metastasis

- signet ring cell carcinoma

Background We report an extremely unusual case of anterior clinoid process (ACP) metastasis as the first presentation of a signet ring cell carcinoma.

Case Description A 54-year-old female patient presented with right-sided visual disturbances due to optic nerve compression from a computed tomography (CT)-identified right anterior clinoid bone lesion. Contrast-enhanced magnetic resonance imaging showed an extra-axial, well-bordered enhancing mass extending from the right ACP toward the inner lumen of the optic canal. Pterional approach was adopted to remove the lesion and decompress the optic canal. Histological examination demonstrated a metastasis from a signet ring cell carcinoma. Postoperative CT showed near-total resection of the tumor and decompression of the optic canal. Visual defect remained unchanged.

Conclusion Metastasis should be considered in the differential diagnosis of the ACP lesions. The early suspicion and identification of this extremely rare pathological entity can be helpful for the prompt management of patients, especially in the absence of any other signs of oncological diseases.
\end{abstract}

\section{Introduction}

The anterior clinoid process (ACP) may be affected by several pathological entities, being meningiomas the most common. ${ }^{1-11}$ Usually, patients harboring ACP lesions present with visual or oculomotor defects because of the involvement of optic nerve, superior orbital fissure, and/or cranial nerves in the cavernous sinus. Anterior clinoidectomy along with tumor removal is the treatment of choice, either performed intra- or extradurally. We herein report the case of a rare case of anterior clinoid metastasis from a silent signet ring cell carcinoma. The very unusual localization and the absence of any other sign of oncological disease made the diagnosis intriguing and difficult to achieve. The aim of this case report is to shed lights on the possibility of considering

received

April 18, 2019

accepted after revision

February 25, 2020 metastatic lesions among the differential diagnosis of pathologies involving the ACP.

\section{Case Description}

A 54-year-old woman presented with 1-month history of visual disturbance in the right eye. Her medical history was unremarkable for other pathologies. She reported positive familiarity for lung adenocarcinoma (father and two brothers). Upon the admission, the computed tomography (CT) scan of the brain showed a hyperdense oval expansive process $(19 \times 14 \mathrm{~mm}$ on the axial plane) of the right ACP, with internal calcifications and bone remodeling ( - Fig. 1). An ophthalmological evaluation demonstrated visual acuity

\footnotetext{
(c) 2020 Georg Thieme Verlag KG Stuttgart · New York
}

License terms

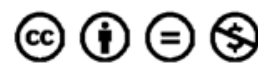




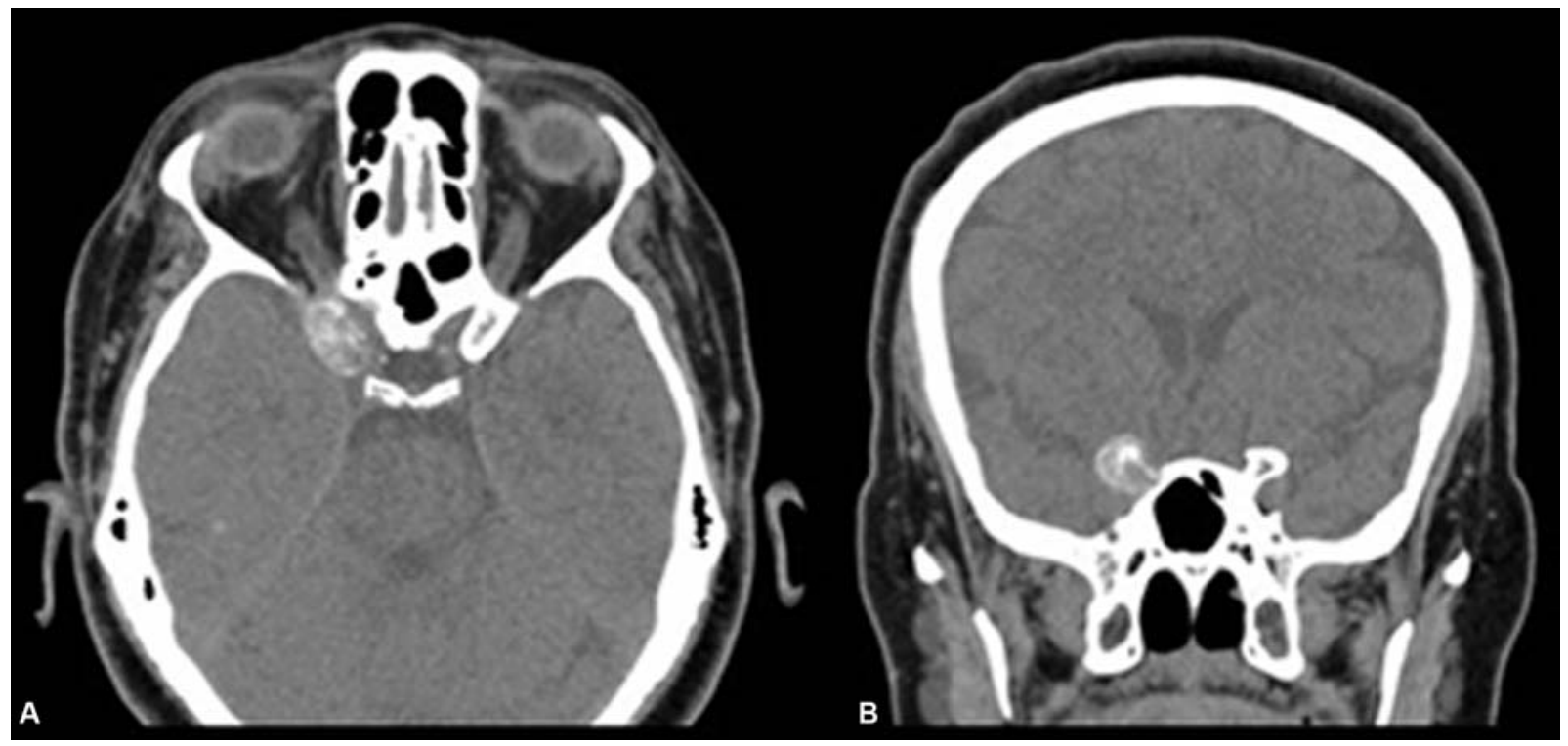

Fig. 1 Preoperative CT scans. Nonenhanced head CT scan, with axial (A) and coronal (B) reformats, demonstrates a lytic lesion of the right anterior clinoid process causing stenosis of the optic canal and marginal involvement of the superior orbital fissure. The lesion appears hyperdense with thin calcifications, representing "sequestra" of normal bone rather than dystrophic calcifications. CT, computed tomography.

of $2 / 10$ and visual field test revealed diffuse reduction of the retinal sensibility, both in the right eye. Contrast-enhanced magnetic resonance imaging showed an extra-axial, well- bordered enhancing mass, extending from the right ACP toward the inner lumen of the optic canal (-Fig. 2 ).

Surgery was suggested to restore the visual function.

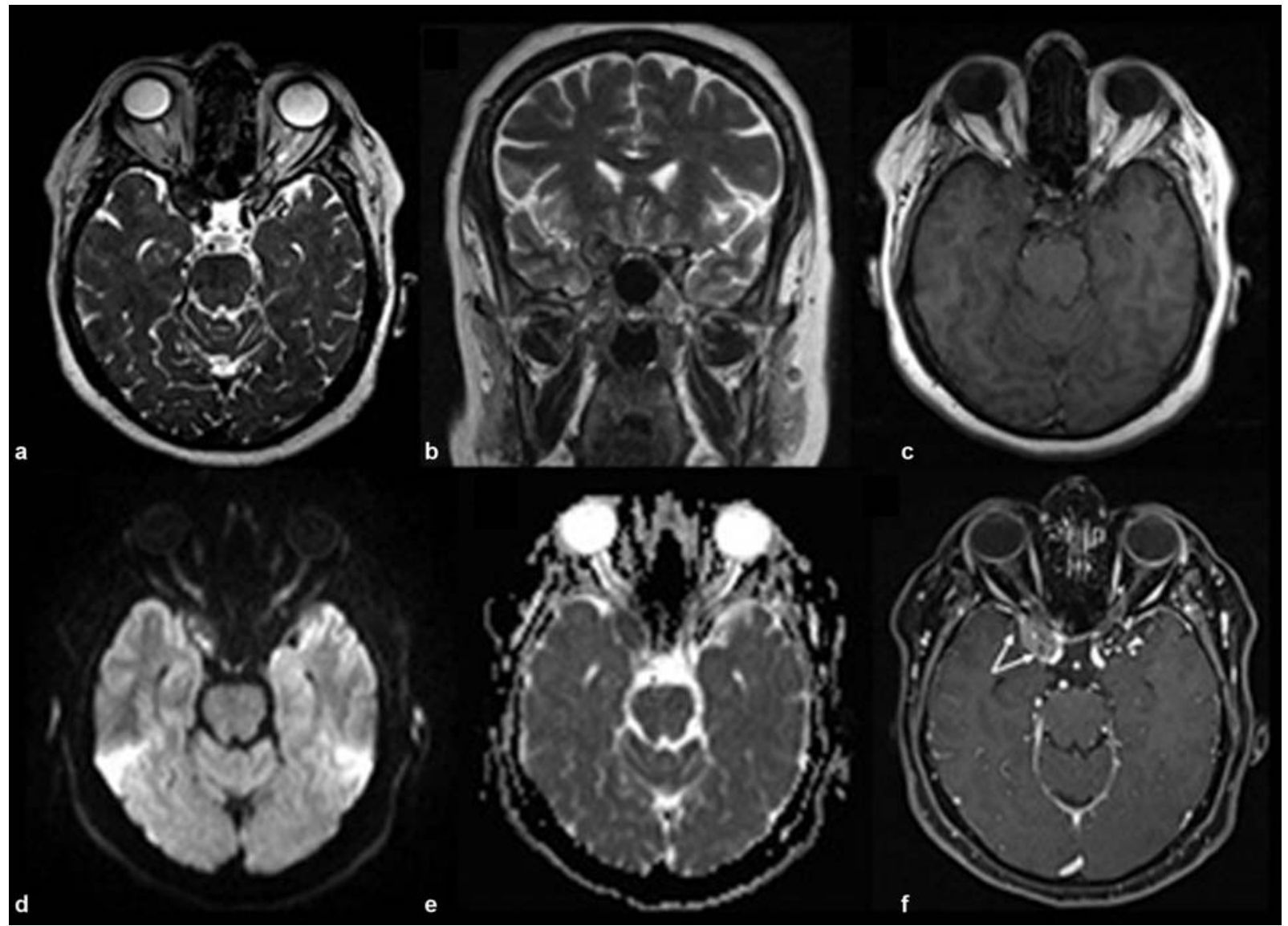

Fig. 2 Preoperative MRI. Contrast-enhanced MRI: axial T2 weighted (A), coronal T2 weighted (B), axial T1 weighted (C), diffusion weighted (b $1,000[D]$ and ADC map [E]), and contrast-enhanced VIBE (F) sequences. MR scan shows an extradural lesion with diffusion restriction (D, E), indicating high cellularity and enhancement. A mild linear dural thickening near the lesion can be also depicted (arrows on F). ADC, apparent diffusion coefficient; MRI, magnetic resonance imaging. 


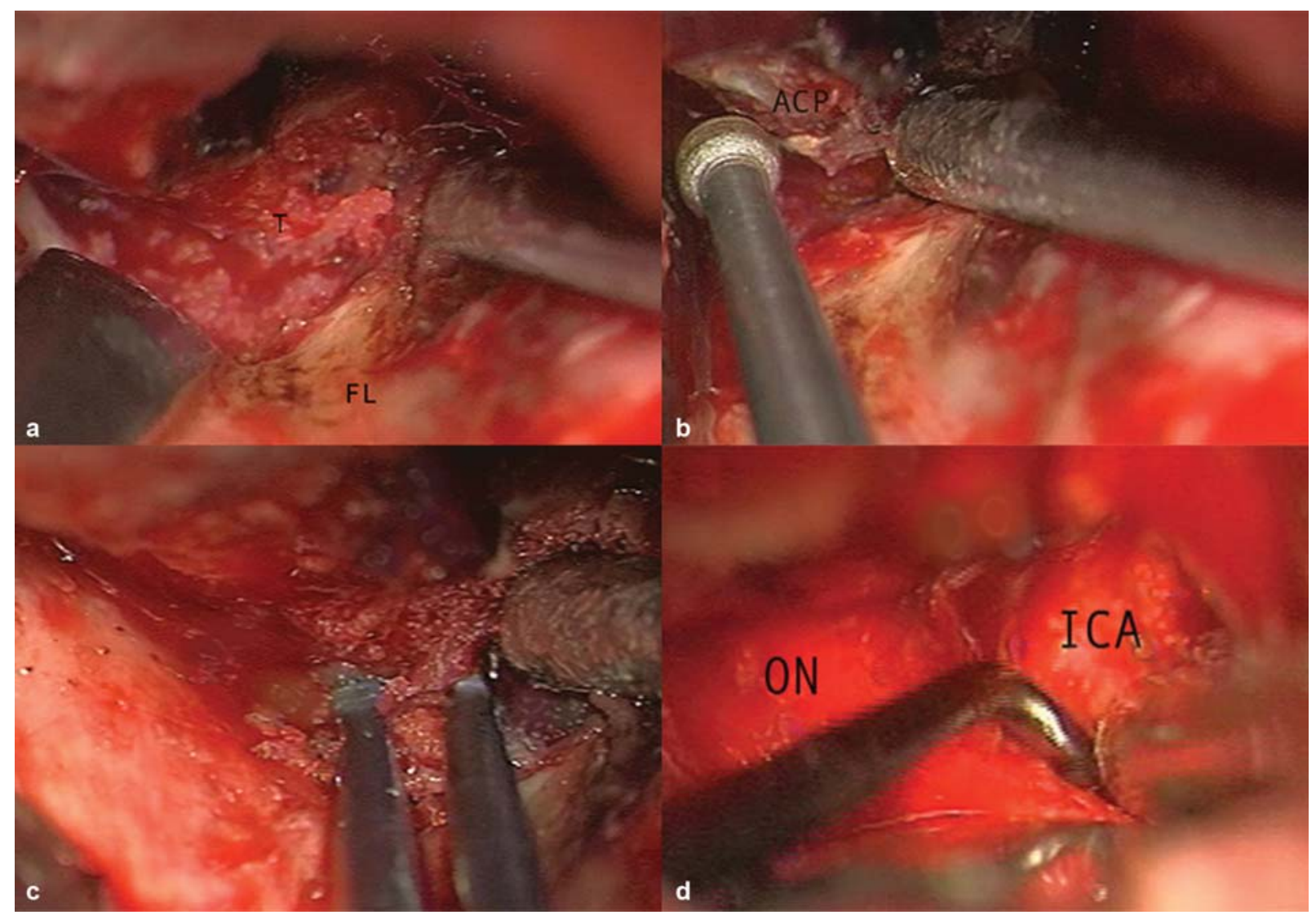

Fig. 3 Intraoperative images. Exposure of the tumor infiltrating the right anterior clinoid process (A). Extradural drilling of the ACP (B). Piecemeal removal of the tumor mass (C). Right optic nerve decompressed and internal carotid artery in the surgical field (D). ACP, anterior clinoid process; FL, frontal lobe; ICA, internal carotid artery; ON, optic nerve; T, tumor.

\section{Surgical Technique}

Right pterional craniotomy was performed and the lesser sphenoid wing was drilled away; the right ACP was reached extradurally and the lesion, which widely infiltrated the clinoid bone, was removed up to the achievement of a satisfactory decompression of the right optic canal-the right optic nerve and its vascularization were seen free of the pathology. Macroscopically, the tumor had reddish-gray soft appearance with calcifications in some regions and it presented a considerable vascularity. At the end of the removal, the dura mater was opened to confirm complete decompression of the optic nerve (-Fig. 3 ).

\section{Pathology}

Histological examination showed an infiltration of fibrous and bone tissue by a neoplasm arranged into nests and ribbons of medium-sized cells characterized by a broad cytoplasm containing large vacuoles full of mucin that displaced the nucleus to the cell's periphery, the typical features observed in case of signet ring cell carcinoma (-Fig. 4). Immunohistochemical evaluation revealed positivity to cytokeratin 7, cytokeratin 20, cytokeratin AE1/3, epithelial membrane antigen ( - Fig. 4), and vimentin. Focal reactivity was observed for CDX2. Progesterone receptor was negative. These findings were highly suggestive of a metastasis from an epithelial neoplasm that, in most cases, originates from the stomach. The major suspect was, there- fore, for a gastric primitive, also because of the positive immunostaining to cytokeratin 7 , cytokeratin 20 , and focal to $\mathrm{CDX} 2$.

\section{Follow-up}

The postoperative CT showed near-total resection of the tumor and decompression of the optic canal ( - Fig. 5). Visual defect remained unchanged. According to tumor board decision, the patient was immediately referred to oncological treatment for radiotherapy and chemotherapy.

\section{Discussion}

The present case exhibits three unusual features: the ACP as extremely rare location for a metastasis, the origin of a suspected gastric cancer-one of the least frequent primary tumors causing intracranial metastases, and the occurrence of a skull base metastasis without any other sign or symptoms. To the best of our knowledge, the present case is the second report on anterior clinoid metastasis in the literature. In the previous case reported by Pojskić et al, the patient was under treatment with chemotherapy for a breast cancer. ${ }^{12}$ Upon the presence of an oncological disease, the occurrence of brain metastasis at the skull base and even at the ACP could be suspected. Albeit frank evidence of a primary disease, meningioma was suspected among the possible differential diagnosis. 


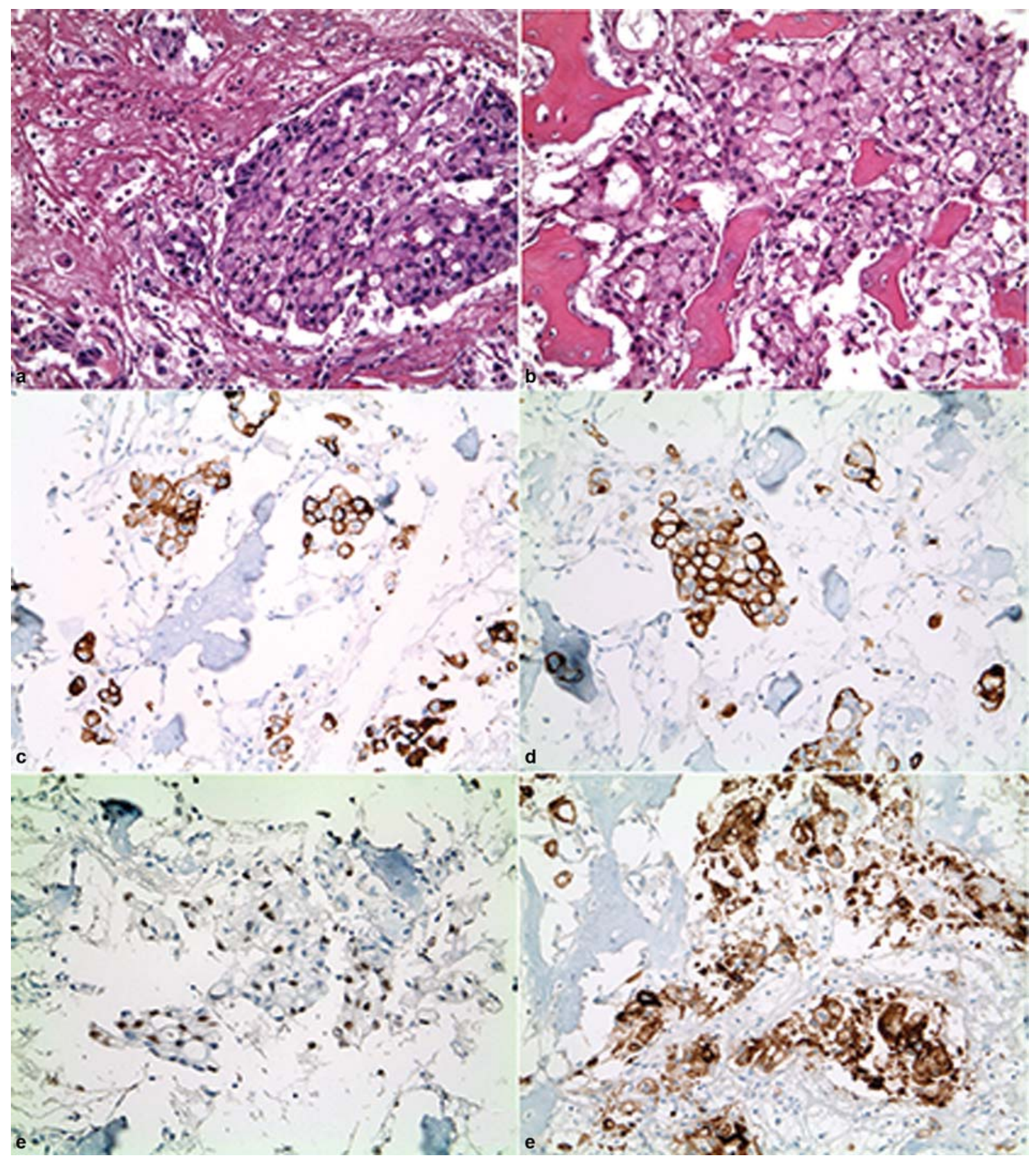

Fig. 4 Histology. The neoplasm was composed by nests of medium-sized cells, characterized by a cytoplasm with clear vacuoles that displace peripherally the nucleus (A, hematoxylin and eosin stain). Infiltration of bone tissue was evident (B, hematoxylin and eosin stain). Immunohistochemistry showed positivity to cytokeratin 7 (C), cytokeratin 20 (D), CDX2 (E) and EMA (F). All pictures were captured at $\times 40$ magnification. EMA, epithelial membrane antigen.

In our case, visual defects were the only presenting signs of an undiagnosed, silent primary tumor; this has made the diagnosis of metastasis for the lesion of the ACP more troublesome. Neuroradiological findings allowed us to exclude common ACP lesions, such as meningiomas and mucoceles, ${ }^{5,13,14}$ favoring the hypothesis of an osseous neoplastic lesion. Indeed, extradural meningiomas are extremely rare, while mucoceles appear as expansile lesions with variable signal on T1- and T2-weighted images (depending on protein concentration) and are characterized by a peripheral linear enhancement. The differential diagnosis included both primary and metastatic bone lesions. Among primary ACP tumor, giant cell tumor has been seldom reported in this location, and therefore, it should be considered. ${ }^{15}$ Other pathologies that seldom may affect the ACP are pyocele, hemangiomas, hemangioblastoma, and dermoid cyst. ${ }^{2,3,7,10}$

Pathology report disclosed a metastasis from a signet ring cell carcinoma, suggestive of a gastric primary tumor, although it has to be said that gastric cancer is one of the least frequent primary tumors causing intracranial metastases. ${ }^{16}$ 


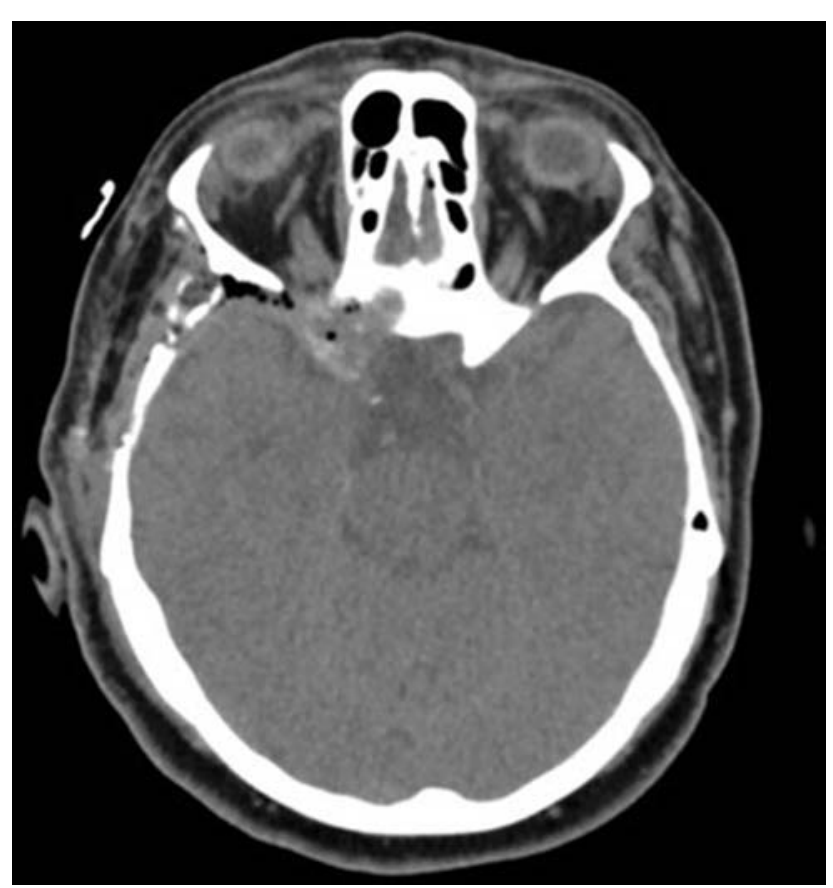

Fig. 5 Early postoperative CT scan. Tumor removal through a right pterional approach and the surgical corridor, partially occupied by air bubbles. CT, computed tomography.

Besides, its presentation as bony metastasis without any gastrointestinal symptom is very unusual and when bone involvement is discovered, tumor usually has reached an advanced metastatic stage. The prognosis of bone metastasis caused by gastric cancer is very poor and the prognosis of patients can be even worse due to a delayed diagnosis. ${ }^{17-23}$

Tumor removal and optic nerve decompression were performed through an extradural clinoidectomy. Extradural removal of the ACP had been extensively described as part of the surgical approach for vascular lesions and skull base tumors. The first reason for performing it extradurally is that dura protects the intradural structures, thus allowing a safer and faster technique. Extradural clinoidectomy allows for a wider bone removal between the optic canal and the superior orbital fissure by providing an easier identification and anatomical orientation than the intradural technique. ${ }^{24-32}$ With the suspicious of a malignant tumor pathology, one more argument favoring the extradural clinoidectomy is to minimize the possibility of intradural tumor spread. ${ }^{12}$ Nonetheless, the long-standing history of the severe visual impairment can explain the lack of any postoperative improvement.

This case highlights the importance of a broad differential diagnosis process, when approaching a patient with bone lesions, with unusual skull base location: the survival period as well as the quality of life may be tremendously improved by making a proper and fast diagnosis and therapy process.

\section{Conclusion}

Metastasis should be considered as possible differential diagnosis of ACP lesions, even in the absence of any sign of oncological disease. Safer and faster removal of ACP metastasis can be accomplished through extradural clinoidectomy. Albeit this pathological entity is extremely rare, the early suspicion supported by neuroradiological findings can be helpful for the prompt management of patients with such unusual clinical features as those described in the present case.

\section{Conflict of Interest}

None declared.

\section{References}

1 Al-Mefty O. Clinoidal meningiomas. J Neurosurg 1990;73(06): 840-849

2 O’Donnell TJ, Michael LM II, Laster R, Fleming JC. Isolated pyocele of anterior clinoid process presenting as a cavernous sinus syndrome. Tenn Med 2013;106(05):37-38, 43

3 Gupta RK, Saran RK, Jagetia A, Narang P. Extra-axial dural cavernous hemangioma with dural tail sign, masquerading as meningioma. J Neurosci Rural Pract 2016;7(04):615-616

4 Morgenstern P, Pisapia D, Ramakrishna R. Calvarial plasmacytoma mimicking meningioma as the initial presentation of multiple myeloma. Cureus 2017;9(03):e1126

5 Aoyama Y, Ohtomo K, Sawamura H. Recurrent optic neuropathy caused by a mucocele of the anterior clinoid process after a 5-year remission: a case report and literature review. Neuroophthalmology 2014;38(05):281-285

6 Chagla AS, Bhaganagare A, Kansal R, Tyagi D. Complete recovery of visual loss following surgical treatment of mucopyocele of the anterior clinoid process. J Clin Neurosci 2010;17(05):670-672

7 Higgins HL, Schimdt JH III. Atypical presentation of a dermoid brain cyst. W V Med J 1996;92(06):312-315

8 Hong CS, Lehman NL, Sauvageau E. A pilocytic astrocytoma mimicking a clinoidal meningioma. Case Rep Radiol 2014;2014:524574

9 Kasliwal MK, Suri A, Gupta DK, Suri V, Rishi A, Sharma BS. Sphenoid wing inflammatory pseudotumor mimicking a clinoidal meningioma: case report and review of the literature. Surg Neurol 2008;70(05):509-513, discussion 513

10 Mansour TR, Medhkour Y, Entezami P, Mrak R, Schroeder J, Medhkour A. The art of mimicry: anterior clinoid dural-based cavernous hemangioma mistaken for a meningioma. World Neurosurg 2017;100:708.e19-708.e22

11 Azarpira N, Noshadi P, Pakbaz S, Torabineghad S, Rakei M, Safai A. Dural plasmacytoma mimicking meningioma. Turk Neurosurg 2014;24(03):403-405

12 Pojskić M, Zbytek B, Arnautović KI. Anterior clinoid metastasis removed extradurally: first case report. J Neurol Surg Rep 2018; 79(02):e55-e62

13 Copeland WR, Van Gompel JJ, Giannini C, Eckel LJ, Koeller KK, Link MJ. Can preoperative imaging predict tumor involvement of the anterior clinoid in clinoid region meningiomas? Neurosurgery 2015;77(04):525-529, discussion 530

14 Watts J, Box G, Galvin A, Brotchie P, Trost N, Sutherland T. Magnetic resonance imaging of meningiomas: a pictorial review. Insights Imaging 2014;5(01):113-122

15 Gelinne A, Akture E, Tranmer B. Tenosynovial giant cell tumor of the clinoid: rare condition. World Neurosurg 2018;118:168-171

16 Go PH, Klaassen Z, Meadows MC, Chamberlain RS. Gastrointestinal cancer and brain metastasis: a rare and ominous sign. Cancer 2011;117(16):3630-3640

17 Lee A, Chang KH, Hong H, Kim H. Sixth cranial nerve palsy caused by gastric adenocarcinoma metastasis to the clivus. J Korean Neurosurg Soc 2015;57(03):208-210

18 Hirai O, Kikuchi H, Hashimoto N. Skull base metastasis from gastric cancer-case report. Neurol Med Chir (Tokyo) 1992;32 (12):908-910 
19 Fukushima M, Katayama Y, Shigemori Y, Miyake H, Hirayama T, Kotani A. Clivus metastasis from gastric signet ring cell carcinoma after a 10-year disease-free interval-case report. Neurol Med Chir (Tokyo) 2012;52(10):751-753

20 Sano T, Aiko T. New Japanese classifications and treatment guidelines for gastric cancer: revision concepts and major revised points. Gastric Cancer 2011;14(02):97-100

21 Nie RC, Yuan SQ Li YF, et al. Clinicopathological characteristics and prognostic value of signet ring cells in gastric carcinoma: a meta-analysis. J Cancer 2017;8(17):3396-3404

22 Mohandas KM, Swaroop VS, Krishnamurthy S, et al. Unusual bone metastasis as the initial symptom of gastric cancer-a report of four cases. Indian J Cancer 1993;30(03):146-150

23 Dittus C, Mathew H, Malek A, Negroiu A. Bone marrow infiltration as the initial presentation of gastric signet ring cell adenocarcinoma. J Gastrointest Oncol 2014;5(06):E113-E116

24 Drake CG, Vanderlinden RG, Amacher AL. Carotid-ophthalmic aneurysms. J Neurosurg 1968;29(01):24-31

25 Noguchi A, Balasingam V, Shiokawa Y, McMenomey SO, Delashaw JB Jr. Extradural anterior clinoidectomy. Technical note. J Neurosurg 2005;102(05):945-950
26 Mariniello G, de Divitiis O, Seneca V, Maiuri F. Classical pterional compared to the extended skull base approach for the removal of clinoidal meningiomas. J Clin Neurosci 2012;19(12):1646-1650

27 Dolenc V. Direct microsurgical repair of intracavernous vascular lesions. J Neurosurg 1983;58(06):824-831

28 Day JD, Giannotta SL, Fukushima T. Extradural temporopolar approach to lesions of the upper basilar artery and infrachiasmatic region. J Neurosurg 1994;81(02):230-235

29 Krisht AF, Kadri PA. Surgical clipping of complex basilar apex aneurysms: a strategy for successful outcome using the pretemporal transzygomatic transcavernous approach. Neurosurgery 2005;56(2, Suppl):261-273, discussion 261-273

30 Andrade-Barazarte $\mathrm{H}$, Jägersberg M, Belkhair S, et al. The extended lateral supraorbital approach and extradural anterior clinoidectomy through a frontopterio-orbital window: technical note and pilot surgical series. World Neurosurg 2017;100:159-166

31 Dolenc VV. A combined epi- and subdural direct approach to carotid-ophthalmic artery aneurysms. J Neurosurg 1985;62(05): 667-672

32 Risi P, Uske A, de Tribolet N. Meningiomas involving the anterior clinoid process. Br J Neurosurg 1994;8(03):295-305 